\title{
Blowing Up at Infinity of Eigenfunctions on Riemannian Manifolds
}

\author{
Yasuyuki FUKAMI \\ Department of Mathematics, Kanazawa University, Kanazawa 920-1192, Japan*
}

Received March 31, 2006; final version accepted June 5, 2006

\begin{abstract}
We study eigenfunctions of a complete, noncompact Riemannian manifold and investigate how they blow up at infinity. Our estimate is also related to the size of the manifold at infinity when the Ricci curvature is nonnegative.
\end{abstract}

KEYWORDS: Riemannian manifold, Laplacian, eigenfunction, Ricci curvature, comparison theorem

\section{Introduction}

Let $M$ be a $m$-dimensional complete noncompact Riemannian manifold, and denote the Laplacian of $M$ by $\Delta_{M}$, where $\Delta_{M}=-$ div grad. In [1] and [2], Borbély studies positive eigenfunctions on $M$ under the condition that the Ricci curvature of $M$ is bounded below by $-(m-1) k^{2},(k \geq 0)$, and shows among other things that a positive eigenfunction $u$ of nonpositive eigenvalue $-\mu$, that is, a positive solution $u$ of equation: $\Delta_{M} u=-\mu u$ satisfies

$$
-\sqrt{\lambda_{k}}+\sqrt{\lambda_{k}+\mu} \leq \sup _{M}|\nabla \log u| \leq \sqrt{\lambda_{k}}+\sqrt{\lambda_{k}+\mu}
$$

where $\lambda_{k}=\frac{(m-1)^{2} k^{2}}{4}$.

The aim of this paper is to investigate how eigenfunctions of negative eigenvalues blow up at infinity.

To state our main results, we fix a point $o$ of $M$ and denote by $B_{r}(o)$ the metric ball of radius $r$ around $o$.

Theorem 1. Let $M$ be a complete noncompact Riemannian manifold of dimension $m$ with Ricci curvature bounded below by $-(m-1) k^{2},(k \geq 0)$.

Let $u$ be an eigenfunction on $M$ with negative eigenvalue $-\mu$. Then

$$
\liminf _{r \rightarrow \infty} \frac{\log \max _{\partial B_{r}(o)}|u|}{r} \geq-\sqrt{\lambda_{k}}+\sqrt{\lambda_{k}+\mu}
$$

where $\lambda_{k}=\frac{(m-1)^{2} k^{2}}{4}$.

In the case where the Ricci curvature of $M$ is nonnegative, we can see a certain relation between the growth of positive eigenfunctions on $M$ and the size of $M$ at infinity. In fact, we prove the following

Theorem 2. Let $M$ be a complete noncompact Riemannian manifold of dimension $m$ with nonnegative Ricci curvature. Suppose that the diameter of the metric sphere $\partial B_{r}(o)$ of radius $r$ around a fixed point $o \in M$ is bounded above by ar for some positive constant $a$ and any large $r$. Let $u$ be a positive eigenfunction of $M$ with negative eigenvalue $-\mu$. Then

$$
\liminf _{r \rightarrow \infty} \frac{\log \min _{\partial B_{r}(o)} u}{r} \geq(1-a) \sqrt{\mu} .
$$

In particular, every positive eigenfunction of negative eigenvalue exponentially blows up at infinity if a 1 .

The outline of the paper is as follows. Section 1 is devoted giving some preliminary results. In Section 2, we will find Theorem 1 still holds true for eigensections of certain Hermitian vector bundles over $M$ (see Theorem 7). Theorem 2 will be proved at the end of Section 2. In Appendix, we review the Sturm-Liouville comparison theorem.

Acknowledgments. The author thanks Professor Atsushi Kasue for his helpful suggestions on the subject of this paper.

\section{Preliminaries}

Let $f(r)$ be a monotone increasing smooth function defined on $[0,+\infty)$ with $f(0)=0$ and $f^{\prime}(0)=1$. Given an integer $m \geq 2$ and a number $\mu>0$, consider the ordinary differential equation:

\footnotetext{
* Current Address: 13-A17-\#405, Umegaoka 1-chome, Neyagawa-shi, Osaka 572-0803, Japan
} 


$$
\phi^{\prime \prime}+(m-1) \frac{f^{\prime}}{f} \phi^{\prime}=\mu \phi
$$

subject to the initial condition:

$$
\phi(0)=1, \quad \phi^{\prime}(0)=0 .
$$

By introducing a differential operator $E_{f}=f^{m-1}(r) \frac{d}{d r}$, the ordinary differential Eq. (2) is equivalent to the following

$$
E_{f}^{2} \phi=\mu f^{2(m-1)} \phi \text {. }
$$

We denote by $\phi_{\mu, f}$ the unique solution of (2) with (3). Then it is easy to see that $\phi_{\mu, f}$ is positive and increasing in $[0,+\infty)$ by the change of variables. In addition, we have

\section{Lemma 3.}

(i) If $\int_{R}^{\infty}\left(\int_{R}^{z} f^{m-1}(y) d y / f^{m-1}(z)\right) d z=\infty$ for some $R>0$, then $\phi_{\mu, f}$ (r) diverges to infinity as $r \rightarrow \infty$.

(ii) If $\int_{R}^{\infty}\left(\int_{R}^{z} f^{m-1}(y) d y / f^{m-1}(z)\right) d z<\infty$ and $\int_{R}^{\infty}\left(1 / f^{m-1}(s)\right) d s<\infty$ for some $R>0$, then $\phi_{\mu, f}(r)$ is bounded as $r \rightarrow \infty$.

Proof. By integrating $\left(2^{\prime}\right)$ from $R$ to $r$, we have

$$
E_{f} \phi_{\mu, f}(r)-E_{f} \phi_{\mu, f}(R)=\mu \int_{R}^{r} \phi_{\mu, f} f^{m-1} d s .
$$

Since $E_{f} \phi_{\mu, f}(R)>0$ and $\phi_{\mu, f}(R) \leq \phi_{\mu, f}(s)$ for $s \geq R$, we get

$$
E_{f} \phi_{\mu, f}(r) \geq \mu \phi_{\mu, f}(R) \int_{R}^{r} f^{m-1} d s,
$$

which shows

$$
\phi_{\mu, f}^{\prime}(r) \geq \mu \phi_{\mu, f}(R) \frac{\int_{R}^{r} f^{m-1} d s}{f^{m-1}(r)}
$$

Thus integrating this, we obtain

$$
\phi_{\mu, f}(r) \geq \phi_{\mu, f}(R)+\mu \phi_{\mu, f}(R) \int_{R}^{r} \frac{\int_{R}^{z} f^{m-1}(s) d s}{f^{m-1}(z)} d z \quad \text { for } r>R
$$

This implies the first assertion (i).

On the other hand, since $\phi_{\mu, f}(R) \leq \phi_{\mu, f}(r)$, we have

$$
E_{f} \phi_{\mu, f}(r)-E_{f} \phi_{\mu, f}(R) \leq \mu \phi_{\mu, f}(r) \int_{R}^{r} f^{m-1} d s,
$$

and hence

$$
\frac{\phi_{\mu, f}^{\prime}(r)}{\phi_{\mu, f}(r)} \leq \frac{E_{f} \phi_{\mu, f}(R)}{\phi_{\mu, f}(r) f^{m-1}(r)}+\mu \frac{\int_{R}^{r} f^{m-1} d s}{f^{m-1}(r)} .
$$

Integrating this, we obtain

$$
\log \frac{\phi_{\mu, f}(r)}{\phi_{\mu, f}(R)} \leq \frac{E_{f} \phi_{\mu, f}(R)}{\phi_{\mu, f}(R)} \int_{R}^{r} \frac{1}{f^{m-1}(s)} d s+\mu \int_{R}^{r} \frac{\int_{R}^{z} f^{m-1}(s) d s}{f^{m-1}(z)} d z,
$$

from which the second assertion (ii) follows.

Given a monotone increasing smooth function $f$ on $[0,+\infty)$ satisfying $f(0)=0$ and $f^{\prime}(0)=1$, we denote by $M_{f}$ the warped product $\mathbf{R}_{\geq 0} \times_{f} S^{m-1}$ with a Riemannian metric $d r^{2}+f^{2}(r) d \theta^{2}$, where $d \theta^{2}$ is the standard metric of the Euclidean unit sphere $S^{m-1}$. Let $r$ be the distance in $M_{f}$ to the pole $o$. Then for a positive number $\mu$, the function $\phi_{\mu, f}(r)$ is an eigenfunction of $M_{f}$ with negative eigenvalue $-\mu$, that is, $\Delta_{M_{f}} \phi_{\mu, f}(r)=-\mu \phi_{\mu, f}(r)$, such that $\phi_{\mu, f}(r)=1$ at $o$. We recall here that the radial curvature (relative to the pole $o$ ) is given by $-f^{\prime \prime}(r) / f(r)$. Given a nonnegative constant $k$, let $f_{k}(r)=r$ for $k=0$ and $f_{k}(r)=k^{-1} \sinh k r$ for $k>0$. Then the warped product $M_{f_{k}}$ of the warping function $f_{k}$ is nothing but the simply connected space form $M\left(-k^{2}\right)$ of constant curvature $-k^{2}$.

Lemma 4. Let $r$ be the distance function to a point of $M\left(-k^{2}\right)$ and $\phi_{\mu, f_{k}}(r)$ the (unique) positive eigenfunction of $M\left(-k^{2}\right)$ (which is rotationally symmetric around o and satisfies $\phi_{\mu, f_{k}}(r)=1$ at o). Then 


$$
\liminf _{r \rightarrow \infty} \frac{\log \phi_{\mu, f_{k}}(r)}{r} \geq-\sqrt{\lambda_{k}}+\sqrt{\lambda_{k}+\mu}
$$

where $\lambda_{k}=\frac{(m-1)^{2} k^{2}}{4}$.

Proof. Let $\widetilde{\phi}(r)=e^{\sqrt{\mu_{\epsilon}} r}$, where $\sqrt{\mu_{\epsilon}}=\left(-\sqrt{\lambda_{k}}+\sqrt{\lambda_{k}+(1+\epsilon) \mu}\right) /(1+\epsilon)$ and $\epsilon$ is a positive constant. Let $\Psi(r)=$ $\phi_{\mu, f_{k}}^{\prime}(r) / \phi_{\mu, f_{k}}(r)$. The function $\Psi(r)$ satisfies the following equation:

$$
\Psi^{\prime \prime}(r)+2 \Psi(r) \Psi^{\prime}(r)+(m-1) \frac{f_{k}^{\prime}(r)}{f_{k}(r)} \Psi^{\prime}(r)+(m-1)\left(\frac{f_{k}^{\prime}(r)}{f_{k}(r)}\right)^{\prime} \Psi(r)=0 .
$$

By the maximal principle, there is no point attaining the maximum of the function $\Psi(r)$. So, comparing with (1), there exists a divergent sequence $\left\{r_{n}\right\}$ satisfying $\lim _{n \rightarrow \infty} \Psi\left(r_{n}\right)=\sup _{r \geq 0} \Psi(r)$. Thus for any $\epsilon>0$ the following inequality holds:

$$
\phi_{\mu, f_{k}}^{\prime}\left(r_{0}\right) / \phi_{\mu, f_{k}}\left(r_{0}\right) \geq \sqrt{\mu_{\epsilon}}\left(=\widetilde{\phi}^{\prime}\left(r_{0}\right) / \widetilde{\phi}\left(r_{0}\right)\right)
$$

for sufficient large $r_{0}>0$.

For any $\epsilon>0$ one can take a sufficient large positive number $r_{0}>0$ in such a way that

$$
-\epsilon \sqrt{\mu_{\epsilon}}<2 \sqrt{\lambda_{k}}-(m-1) f_{k}^{\prime}(r) / f_{k}(r)<\epsilon \sqrt{\mu_{\epsilon}}
$$

for any $r \geq r_{0}$. Then direct computations show that

$$
\begin{aligned}
E_{f_{k}}^{2} \widetilde{\phi}(r) & =\mu_{\epsilon}\left(1+(m-1) \frac{f_{k}^{\prime}(r)}{\sqrt{\mu_{\epsilon}} f_{k}(r)}\right) f_{k}^{2(m-1)}(r) \widetilde{\phi}(r) \\
& \leq \mu_{\epsilon}\left(1+2 \sqrt{\frac{\lambda_{k}}{\mu_{\epsilon}}}+\epsilon\right) f_{k}^{2(m-1)}(r) \widetilde{\phi}(r)=\mu f_{k}^{2(m-1)}(r) \widetilde{\phi}(r)
\end{aligned}
$$

for all $r \geq r_{0}$.

These inequalities allow us to apply the Sturm-Liouville comparison theorem (see Lemma 10 in Appendix) to $\phi_{\mu, f_{k}}(r)$ and $\tilde{\phi}(r)$, and obtain $\phi_{\mu, f_{k}}(r) / \phi_{\mu, f_{k}}\left(r_{0}\right) \geq \widetilde{\phi}(r) / \widetilde{\phi}\left(r_{0}\right)$ for all $r \geq r_{0}$. As a result, we have $\liminf { }_{r \rightarrow \infty}\left(\log \phi_{\mu, f_{k}}(r)\right) / r \geq \sqrt{\mu_{\epsilon}}$, and letting $\epsilon$ tend to 0 , we thus attain Lemma 4 .

Lemma 5. Let $M$ be a complete noncompact Riemannian manifold of dimension $m$ and $r$ the distance function to a fixed point o of M. Suppose that the Ricci curvature of $M$ is bounded below by a function $-(m-1) f^{\prime \prime}(r) / f(r)$, where $f(r)$ is a positive smooth function on $[0,+\infty)$ such that $f(0)=0, f^{\prime}(0)=1$ and $f^{\prime}(r) \geq 0$ on $[0,+\infty)$. Given a positive number $\mu$, let $\phi_{\mu, f}$ be the solution of Eq. (2) subject to the initial condition (3). Then the function $\phi_{\mu, f}(r)$ satisfies

$$
\Delta_{M} \phi_{\mu, f}(r) \geq-\mu \phi_{\mu, f}(r)
$$

on $M$ as a distribution. Moreover if a continuous function $u$ on $M$ satisfies $\Delta_{M}|u| \leq-\mu|u|$ as a distribution, then

$$
\max _{x \in \partial B_{r}(o)}|u|(x) \geq|u(o)| \phi_{\mu, f}(r)
$$

for all $r>0$.

Proof. Since $\phi_{\mu, f}(r)$ is monotone increasing, we can deduce from the Laplacian comparison theorem (see [7], Proposition 2.15) that

$$
\Delta_{M} \phi_{\mu, f}(r) \geq \Delta_{M_{f}} \phi_{\mu, f}(r)=-\mu \phi_{\mu, f}(r) .
$$

In fact this holds everywhere on $M$ as a distribution (cf. [4], [5]).

Let $u$ be as above and set $w=|u|-|u(o)| \phi_{\mu, f}(r)$. Then we have $\Delta_{M} w \leq-\mu w$ as a distribution on $M$. Hence the strong maximal principle for a weak sub-solution (see for example [6] and its final remark) allows us to conclude that $\max _{x \in \partial B_{r}(o)} w=\sup _{x \in B_{r}(o)} w \geq w(o)=0$. This shows that

$$
\max _{x \in \partial B_{r}(o)}|u|(x) \geq|u(o)| \phi_{\mu, f}(r)
$$

for all $r>0$.

\section{A generalization of Theorem 1 and Proof of Theorem 2}

In this section, we first prove Theorem 1 in a more general situation and add some observations. Theorem 2 will be verified at the end of the section.

Let us condsider a Hermitian vector bundle $E$ of rank $r$ over $M$ endowed with a metric connection $\nabla$. We denote by $\Gamma(E)$ the set of smooth sections of $E$ over $M$ and by $\langle\cdot, \cdot\rangle$ the fiberwise Hermitian inner product. And we write 
$|s|(x)=\sqrt{\langle s, s\rangle(x)},(x \in M)$ for sections $s \in \Gamma(E)$. Let $\nabla^{*}$ be the formal adjoint operator of $\nabla$. Given a symmetric endomorphism $R$ of $E$, we define an operator acting on $\Gamma(E)$ by $L=\nabla^{*} \nabla+R$.

Recall Kato's inequality for sections of $E$ due to Braverman, Milatovich and Shubin ([3], Theorem 5.7) in the following:

Lemma 6. Every section $s \in \Gamma(E)$ satisfies

$$
\Delta_{M}|s| \leq \operatorname{Re}\left\langle\nabla^{*} \nabla s, \operatorname{Sign}(s)\right\rangle
$$

as a distribution on $M$, where $\operatorname{Sign}(s)(x)$ is $s(x) /|s(x)|$ for $s(x) \neq 0$ and otherwise 0 .

Now Theorem 1 is extended to the following:

Theorem 7. Let $M$ be a complete noncompact Riemannian manifold of dimension $m$ and $E$ a Hermitian vector bundle with a metric connection $\nabla$ and a symmetric endomorphism $R$ of $E$. Suppose that the Ricci curvature of $M$ is bounded below by $-(m-1) k^{2}$ for some constant $k \geq 0$, and $R$ satisfies $\langle R s, s\rangle \geq K|s|^{2}$ for some constant $K$ and any $s \in \Gamma(E)$. Let $s$ be a nontrivial eigensection of $E$ with eigenvalue $-\mu$, that is, $L s=-\mu s$. If $\mu+K>0$, then

$$
\liminf _{r \rightarrow \infty} \frac{\log \max _{\partial B_{r}(o)}|s|}{r} \geq-\sqrt{\lambda_{k}}+\sqrt{\lambda_{k}+(\mu+K)}
$$

where $o$ is a fixed point of $M$.

Proof. Let $r$ be the distance to a fixed point $o$ of $M$ and set $w=|s|-|s(o)| \phi_{\mu+K, f_{k}}(r)$, where we may assume that $s(o) \neq 0$. Then in view of Lemma 6 , we see that $\Delta_{M} w \leq-(\mu+K) w$ as a distribution on $M$, and hence the assertion follows from Lemmas 4 and 5.

Remark. We consider the case where $E$ is the trivial bundle $M \times \mathbf{C}$ over $M$. Let $A$ be a real-valued 1-form on $M$ and $V$ a real-valued function on $M$. Define a magnetic Laplacian by $\Delta_{M, A}=d_{A}^{*} d_{A}$, where $d_{A}=d-\sqrt{-1} A$ and $d_{A}^{*}$ is its formal adjoint. For any nontrivial solution $u$ of equation $\Delta_{M, A} u+V u=-\mu u$ with a constant $\mu$ such that $\mu+$ $\inf _{M} V>0$, we have

$$
\liminf _{r \rightarrow \infty} \frac{\log \max _{\partial B_{r}(o)}|u|}{r} \geq-\sqrt{\lambda_{k}}+\sqrt{\lambda_{k}+\mu+\inf _{M} V}
$$

where $o$ is a fixed point of $M$. Theorem 1 corresponds to the case where $A=0$ and $V=0$.

Now we consider a complete Riemannian manifold $M$ of dimension $m$ which is minimally immersed into a simply connected, complete Riemannian manifold $H$ with sectional curvature bounded above by $-k_{1}^{2}$. Assume that the Ricci curvature of $M$ is bounded below by $-(m-1) k_{2}^{2}\left(k_{2} \geq k_{1}>0\right)$. Let $b_{\gamma}$ be the Busemann function of $H$ associated with a ray $\gamma$ starting from a point $o \in H$, that is, $b_{\gamma}(x)=\lim _{t \rightarrow \infty}\left\{d_{H}(x, \gamma(t))-t\right\}, x \in H$. Recall the fact that the Hessian of $b_{\gamma}$ satisfies

$$
\nabla d b_{\gamma} \geq k_{1}\left(g_{H}-d b_{\gamma} \otimes d b_{\gamma}\right)
$$

(cf. Theorem A in [7]), and hence we have

$$
\nabla d e^{k_{1} b_{\gamma}} \geq k_{1}^{2} e^{k_{1} b_{\gamma}} g_{H}
$$

where $g_{H}$ denotes the metric of $H$. Let $j: M \rightarrow H$ be a minimal immersion. Then $e^{k_{1} b_{\gamma} \circ j}$ satisfies

$$
\Delta_{M} e^{k_{1} b_{\gamma} \circ j} \leq-k_{1}^{2} m e^{k_{1} b_{\gamma} \circ j}
$$

in $M$. Thus by the same reason as in Theorem 7 , we have

$$
\liminf _{r \rightarrow \infty} \frac{\max _{x \in \partial B_{r}(o)} k_{1} b_{\gamma}(j(x))}{r} \geq-\sqrt{\lambda_{k_{2}}}+\sqrt{\lambda_{k_{2}}+k_{1}^{2} m}>0 .
$$

Since $b_{\gamma}(p) \leq d_{H}(o, p)$ for all $p \in H$, we have thus proved the following:

Proposition 8. Let $j: M \longrightarrow H$ be a minimal immersion of a complete noncompact Riemannian manifold $M$ of dimension $m$ into a simply connected, complete Riemannian manifold $H$ whose sectional curvature is bounded above by $-k_{1}^{2}$. If the Ricci curvature of $M$ is bounded below by $-(m-1) k_{2}^{2}$ for some constant $k_{2}\left(\geq k_{1}>0\right)$, then

$$
\liminf _{r \rightarrow \infty} \frac{\max _{x \in \partial B_{r}(o)} d_{H}(j(x), o)}{r} \geq \frac{-\sqrt{\lambda_{k_{2}}}+\sqrt{\lambda_{k_{2}}+k_{1}^{2} m}}{k_{1}},
$$

where $d_{H}(j(x), o)$ is the distance in $H$ between $j(x)$ and a fixed point of $H$.

We note that the function $e^{k_{1} b_{\gamma} \circ j}$ as above is a positive eigenfunction of $M$ with eigenvalue $-k_{1}^{2} m$ in the case where $H=M\left(-k_{1}^{2}\right)$, that is, $H$ is the simply connected hyperbolic space form of constant curvature $-k_{1}^{2}$. We also remark that if $H$ has nonpositive sectional curvature, then 


$$
\max _{x \in \partial B_{r}(o)} d_{H}(j(x), o) \geq C \sqrt{r}
$$

for some positive constant $C$ and all sufficient large $r$. See Proposition 2 in [8] for the proof. In addition, relevantly to the subject of [8], we have the following:

Proposition 9. Let $M$ be a complete noncompact Riemannian manifold of dimension $m$ and suppose that the Ricci curvature is bounded below by $-c\left(r^{2}+1\right)$ for some positive constant $c$, where $r$ is the distance to a fixed point of M. Let $u$ be a nontrivial continuous function on $M$ satisfying $\Delta_{M}|u| \leq-\mu|u|$ with a negative constant $-\mu$ as a distribution. Then $u$ is an unbounded function on $M$.

Proof. Let $f$ be the solution of equation: $f^{\prime \prime}(r)-c\left(r^{2}+1\right) f(r)=0$, subject to the conditions: $f(0)=0$ and $f^{\prime}(0)=1$. Then in view of Lemmas 3 and 5, it suffices to verify that

$$
\frac{\int_{R}^{r} f^{m-1}(s) d s}{f^{m-1}(r)} \geq \frac{C}{r} \quad(\text { on }(R,+\infty))
$$

for some constant $C$ and $R$.

To see this, let $\psi_{f}(r)=f^{\prime}(r) / f(r)$ and $\eta(r)=A r$, where $A$ is a positive constant chosen in such a way that $A \geq \max \left\{\sqrt{c}, c, \psi_{f}(R) / R\right\}$. Then obviously $\eta(R) \geq \psi_{f}(R)$ and also

$$
\eta^{\prime}(r)+\eta(r)^{2} \geq A^{2} r^{2}+A \geq c r^{2}+c=\psi_{f}^{\prime}(r)+\psi_{f}(r)^{2} .
$$

Therefore it follows from the classical comparison theorem on Riccati equation that

$$
\psi_{f}(r) \leq \eta(r)=A r \quad \text { for all } r \geq R .
$$

On the other hand, direct calculus shows that

$$
\frac{\int_{R}^{r} f^{m-1}(s) d s}{f^{m-1}(r)}=\frac{1}{(m-1) \psi_{f}\left(\xi_{r}\right)}\left(1-\frac{\int_{0}^{R} f^{m-1}(s) d s}{\int_{0}^{r} f^{m-1}(s) d s}\right)
$$

for some $0<\xi_{r}<r$. Combining (5) and (6), we get the inequality (4) mentioned above.

Proof of Theorem 2. For any $r>0$, take points $x, y \in \partial B_{r}(p)$ satisfying $u(x)=\max _{\partial B_{r}(p)} u$ and $u(y)=\min _{\partial B_{r}(p)} u$. Then by Borbély's estimate (1) and integrating the gradient of $\log u$ along a minimizing path joining $x$ to $y$, we get $u(x) \leq e^{\sqrt{\mu} d(x, y)} u(y)$. By the assumption on the diameter of the metric sphere $\partial B_{r}(o)$, we see that $u(x) \leq e^{a \sqrt{\mu} r} u(y)$. On the other hand, in view of Theorem 1 , for any $\epsilon>0$, there exists a constant $C>0$ such that $u(x) \geq C e^{\sqrt{\mu}(1-\epsilon) r}$ for all sufficient large $r$. Hence we have $\min _{\partial B_{r}(p)} u \geq C e^{(1-a-\epsilon) \sqrt{\mu} r}$, from which it follows that $\liminf _{r \rightarrow \infty}\left(\log \min _{\partial B_{r}(o)} u\right) / r \geq$ $(1-a-\epsilon) \sqrt{\mu}$. Letting $\epsilon$ tend to 0 , we obtain Theorem 2 .

\section{Appendix}

We show the Sturm-Liouville comparison theorem with respect to the operator $E_{f}=f^{m-1}(r) \frac{\partial}{\partial r}$.

Lemma 10. Let $f(r)$ be a monotone increasing $C^{2}$-function on $[0,+\infty)$ with $f(0)=0$. Let $u$, $v$ be respectively positive $C^{2}$-functions satisfying

$$
\begin{aligned}
& E_{f}^{2} u(r) \geq P(r) u(r), \\
& E_{f}^{2} v(r) \leq Q(r) v(r)
\end{aligned}
$$

on $\left[r_{0},+\infty\right)$ for some $r_{0}>0$. If $u^{\prime}\left(r_{0}\right) / u\left(r_{0}\right) \geq v^{\prime}\left(r_{0}\right) / v\left(r_{0}\right)$ and $P(r) \geq Q(r)$ for all $r \geq r_{0}$, then

$$
\frac{u(r)}{u\left(r_{0}\right)} \geq \frac{v(r)}{v\left(r_{0}\right)} \quad \text { for } r \geq r_{0} .
$$

Proof. Noting that $E_{f}(u / v)=\left\{E_{f} u \cdot v-u \cdot E_{f} v\right\} / v^{2}$, we have

$$
\begin{aligned}
E_{f}\left\{E_{f} u \cdot v-u \cdot E_{f} v\right\} & =E_{f}^{2} u \cdot v+E_{f} u \cdot E_{f} v-E_{f} u \cdot E_{f} v-u \cdot E_{f}^{2} v \\
& =(P-Q) u v \geq 0 .
\end{aligned}
$$

As a result, $E_{f} u \cdot v-u \cdot E_{f} v$ is an increasing function, and hence $\left(E_{f} u \cdot v-u \cdot E_{f} v\right)(r) \geq\left(E_{f} u \cdot v-u \cdot E_{f} v\right)\left(r_{0}\right) \geq 0$ for all $r \geq r_{0}$. This shows that $E_{f}(u / v) \geq 0$ on $\left[r_{0},+\infty\right)$. Thus we have $u(r) / v(r) \geq u\left(r_{0}\right) / v\left(r_{0}\right)$ if $r \geq r_{0}$. 


\section{REFERENCES}

[1] Borbély, A., "Sharp gradient estimates for eigenfunctions on Riemannian manifolds," Bull. Austral. Math. Soc., 57: 253-259 (1998).

[2] Borbély, A., "Gradient comparison theorems for harmonic functions on Riemannian manifolds," Quart. J. Math. Oxford Ser., 51: 19-37 (2000).

[3] Braverman, M., Milatovich, O., and Shubin, M., "Essential selfadjointness of Schrödinger-type operators on manifolds, translation in Russian Math.," Surveys, 57: 641-692 (2002).

[4] Cheeger, J., and Yau, S. T., “A lower bound for the heat kernel," Comm. Pure Appl. Math., 34: 465-480 (1981).

[5] Cheng, S. Y., "Eigenvalue comparison theorems and its geometric applications," Math. Z., 143: $289-297$ (1975).

[6] Friedman, A., "A strong maximum principle for weakly subparabolic functions," Pacific J. Math., 11: 175-184 (1961).

[7] Greene, R. E., and Wu, H., "Function theory on manifolds which possess a pole," Lecture Notes in Mathematics, 699: Springer, Berlin, 1979.

[8] Kasue, A., Estimates for solutions of Poisson equations and their applications to submanifolds, Differential Geometry of Submanifolds (Kyoto, 1984), 1-14, Lecture Notes in Mathematics, 1090, Springer, Berlin, 1984. 